Louisiana State University

LSU Digital Commons

Faculty Publications

Department of Mathematics

$10-1-2021$

\title{
Stability Analysis Using Generalized Sup-Delay Inequalities
}

Frederic Mazenc

Laboratoire des Signaux et Systèmes

Michael Malisoff

Louisiana State University

Miroslav Krstic

University of California, San Diego

Follow this and additional works at: https://digitalcommons.Isu.edu/mathematics_pubs

\section{Recommended Citation}

Mazenc, F., Malisoff, M., \& Krstic, M. (2021). Stability Analysis Using Generalized Sup-Delay Inequalities. IEEE Control Systems Letters, 5(4), 1411-1416. https://doi.org/10.1109/LCSYS.2020.3038367

This Article is brought to you for free and open access by the Department of Mathematics at LSU Digital Commons. It has been accepted for inclusion in Faculty Publications by an authorized administrator of LSU Digital Commons. For more information, please contact ir@lsu.edu. 
archives-ouvertes

\title{
Stability Analysis using Generalized Sup-Delay Inequalities
}

\author{
Frederic Mazenc, Michael Malisoff, Miroslav Krstic
}

\section{To cite this version:}

Frederic Mazenc, Michael Malisoff, Miroslav Krstic. Stability Analysis using Generalized Sup-Delay Inequalities. ACC 2021 - American Control Conference, May 2021, New Orleans, United States. hal-03431315

\section{HAL Id: hal-03431315 https://hal.inria.fr/hal-03431315}

Submitted on 16 Nov 2021

HAL is a multi-disciplinary open access archive for the deposit and dissemination of scientific research documents, whether they are published or not. The documents may come from teaching and research institutions in France or abroad, or from public or private research centers.
L'archive ouverte pluridisciplinaire HAL, est destinée au dépôt et à la diffusion de documents scientifiques de niveau recherche, publiés ou non, émanant des établissements d'enseignement et de recherche français ou étrangers, des laboratoires publics ou privés. 


\title{
Stability Analysis using Generalized Sup-Delay Inequalities
}

\author{
Frederic Mazenc, Michael Malisoff, and Miroslav Krstic
}

\begin{abstract}
We provide a new generalization of a recent trajectory-based approach for proving global asymptotic stability properties, using new sup-delay inequalities. Our generalization eliminates the usual contractivity requirement from previous sup-delay inequality approaches. We show how the requirements of our generalization hold under less restrictive Halanay's conditions than those that were previously reported. While the usual Halanay's inequality requires the decay rate to exceed the gain, our less restrictive conditions allow the gain to exceed the decay rate. We apply our work to systems with switching delays and to a continuous-discrete system.
\end{abstract}

\section{INTRODUCTION}

The stability analysis of systems with delays is a central problem that is motivated by communication delays, gestation delays in biological systems, and delayed information gathering, which commonly arise in engineering systems; see [?], [?], [?], [?], [?], [?], and [?]. Standard approaches to coping with delays entail finding Lyapunov-Krasovskii or Razhumikin functions, which are useful for switched systems [?]. This motivated a literature on building LyapunovKrasovskii or Razhumikin functions for delayed systems, e.g., [?] and [?]. A useful approach to their construction entails transforming strict Lyapunov functions for the systems that are obtained by setting all of the delays to zero.

However, finding strict Lyapunov functions can be difficult. This motivated the search (which began in [?], [?], [?], and [?]) for trajectory-based methods (based on sup-delay inequalities) to prove asymptotic stability properties in cases that are not amenable to traditional Lyapunov approaches, such as continuous-discrete and switched systems; see [?] and [?]. Whereas prior works assumed contractivity conditions of the form $v(t) \leq \kappa \sup _{\ell \in[t-T, t]} v(\ell)$ for all $t \geq 0$ for given piecewise continuous functions $v:[-T,+\infty) \rightarrow$ $[0,+\infty)$ and constants $T>0$ and $\kappa \in(0,1)$, here we relax this contractivity condition to estimates of the form

$$
v(t) \leq \kappa \sup _{\ell \in[t-T, t]} v(\ell)+\rho(t) \sup _{\ell \in[t-\bar{\tau}, t-\underline{\tau}]} v(\ell)+\eta(t)
$$

for given piecewise continuous functions $v$ and $\eta$, constants $T>0, \kappa \in(0,1), \underline{\tau}>0$, and $\bar{\tau}>\underline{\tau}$, and nonnegative valued functions $\rho$ that can take values outside of the interval $(0,1)$.

Supported in part by NSF Grants 1711299 (Malisoff) and 1711373 (Krstic).

F. Mazenc is with EPI DISCO INRIA-Saclay, Laboratoire des Signaux et Systèmes (L2S, UMR CNRS 8506), CNRS, CentraleSupélec, Université Paris-Sud, 3 rue Joliot Curie, 91192, Gif-sur-Yvette, France (email: frederic.mazenc@12s.centralesupelec.fr).

M. Malisoff is with the Department of Mathematics, Louisiana State University, Baton Rouge, LA 70803-4918, USA (email: malisoff@1su.edu).

M. Krstic is with the Department of Mechanical and Aerospace Engineering, University of California, San Diego, La Jolla, CA 92093-0411, USA (email: krstic@ucsd.edu).
This lets us prove input-to-state stability (or ISS) properties of $v$ with respect to $\eta$, and in the special case where $\eta=$ 0 , it proves asymptotic convergence properties under less restrictive versions of Halanay's condition of the form

$$
\dot{v}(t) \leq-a v(t)+c(t) \sup _{\ell \in[t-T, t]} v(\ell)
$$

for constants $a>0$ and $T>0$ and functions $c(t)$ that can take some of their values in $[a,+\infty)$. Due to the preceding strong motivation for generalized Halanay's conditions, our Halanay's conditions in this work are a significant innovation, because of their less restrictive conditions on $c(t)$. See also [?], [?], [?], and [?] for complementary asymptotic stability results under variants of Halanay's conditions.

In Section II, we prove our new stability theorem under our conditions (1). Our proof of our theorem is based on the contractivity lemma from [?], and a new sup-delay inequality lemma where the term $\kappa \sup _{\ell \in[t-T, t]} v(\ell)$ from the contraction approach is replaced by a term of the form $\rho(t) \sup _{\ell \in[t-\bar{\tau}, t-\underline{\tau}]} v(\ell)$ as explained above; for clarity and completeness, we state and prove these lemmas in Appendix A below, and then we use the lemmas to prove our theorem in Appendix B. In Section III, we illustrate how our assumptions hold under Halanay's conditions of the form (2), including cases where the assumptions of the Halanay's inequality results of [?] are not satisfied, and we provide applications to systems with switching delays and continuous-discrete systems. We close in Section IV with a summary of our contributions and planned research.

We use standard notation, which is simplified when no confusion would arise. The standard Euclidean and induced matrix norms are denoted by $|\cdot|$, and $|\cdot|_{S}$ is the supremum over any set $S$. We use $f\left(t^{-}\right)$and $f\left(t^{+}\right)$to denote left and right limits, respectively. By piecewise continuity of a function $f$ on an interval $[f,+\infty)$, we mean that there is a strictly increasing unbounded sequence $\left\{t_{i}\right\}$ such that $t_{0}=f, f$ is continuous on $\left(t_{i}, t_{i+1}\right)$ for each $i$, and $f\left(t^{+}\right)$ and $\bar{f}\left(s^{-}\right)$are finite for all $t \geq \underline{f}$ and $s>\underline{f}$. We set $\mathbb{N}=\{1,2, \ldots\}$ and $\mathbb{Z}_{\geq 0}=\mathbb{N} \cup\{0\}, A \leq B$ for square matrices of the same size means that $B-A$ is nonnegative definite, and $I$ is the identity matrix in the dimension under consideration.

\section{MAIN THEOREM}

To state our theorem, we introduce the following additional notation that will be used throughout this paper. Let $\rho:[0,+\infty) \rightarrow[0,+\infty)$ be a piecewise continuous function such that there are constants $\bar{\rho}>0, \underline{\tau}>0$, and $\bar{\tau} \geq \underline{\tau}$, an integer $p \in \mathbb{N}$, and a constant $\omega \in(0,1)$ such that

$$
\rho(t) \leq \bar{\rho} \text { for all } \mathrm{t} \geq 0
$$


and such that

$$
\prod_{j=0}^{p} \sup _{s \in[\underline{\tau}, \bar{\tau}]} \rho(t-j s) \leq \omega
$$

holds for all $t \geq p \bar{\tau}$. In terms of the constant

$$
\Omega=\omega+\epsilon \sum_{i=0}^{p} \bar{\rho}^{i},
$$

we prove the following result in Appendix B:

Theorem 1: Let $\epsilon \in(0,1)$ be a constant, and $v$ : $[-\bar{\tau},+\infty) \rightarrow[0,+\infty)$ and $\eta:[0,+\infty) \rightarrow[0,+\infty)$ be locally bounded piecewise continuous functions such that

$$
v(t) \leq \rho(t) \sup _{\ell \in[t-\bar{\tau}, t-\underline{\tau}]} v(\ell)+\epsilon \sup _{\ell \in[t-\underline{\tau}, t]} v(\ell)+\eta(t)
$$

is satisfied for all $t \geq 0$, and assume that $\Omega \in(0,1)$. Then

$$
v(t) \leq|v|_{\left[t_{0}-(p+1) \bar{\tau}, t_{0}\right]} e^{\frac{\ln (\Omega)}{(p+1) \bar{\tau}}\left(t-t_{0}\right)}+\frac{\sum_{i=0}^{p} \bar{\rho}^{i}}{1-\Omega}|\eta|_{\left[t_{0}-p \bar{\tau}, t\right]}
$$

holds for all $t \geq t_{0}$ and $t_{0} \geq p \bar{\tau}$.

Remark 1: An estimate of the rate of convergence of $v$ is $-\ln (\Omega) /((p+1) \bar{\tau})$, which depends on $p$ and $\omega$. An interesting open question is whether under our assumptions, one can find $p$ values that give the largest value for this convergence rate. This question may be the subject of future research.

Remark 2: Theorem 1 is no longer if we relax its condition $\epsilon \in(0,1)$ to allow $\epsilon \geq 1$, since the $\epsilon \geq 1$ case allows unbounded $v$ 's; see Remark A.5 in Appendix A.

Remark 3: To see how our conditions on $\rho$ can be satisfied for a continuous function $\rho$ that takes some values in $(1,+\infty)$, consider the case where $\underline{\tau}=\frac{\pi}{2}, \bar{\tau}=\frac{\pi}{2}+\frac{1}{10}$, and $\rho(t)=\frac{9}{8} \sin ^{2}(t)$. Then

$$
\begin{aligned}
\prod_{j=0}^{1} \sup _{s \in[\underline{\tau}, \bar{\tau}]} \rho(t-j s) & =\frac{81}{64} \sin ^{2}(t) \sup _{s \in\left[\frac{\pi}{2}, \frac{\pi}{2}+\frac{1}{10}\right]} \sin ^{2}(t-s) \\
& =\frac{81}{64} \sin ^{2}(t) \sup _{s \in\left[0, \frac{1}{10}\right]} \cos ^{2}(t-s)
\end{aligned}
$$

for all $t \geq 0$. Since $|\cos (t-s)| \leq|\cos (t)|+\frac{1}{10}$ for all $s \in\left[0, \frac{1}{10}\right]$, we deduce that

$$
\begin{aligned}
\prod_{j=0}^{1} \sup _{s \in[\underline{\tau}, \bar{\tau}]} \rho(t-j s) & \leq \frac{81}{64} \sin ^{2}(t)\left[|\cos (t)|+\frac{1}{10}\right]^{2} \\
& =\frac{81}{64}\left[|\sin (t)||\cos (t)|+\frac{1}{10}\right]^{2} .
\end{aligned}
$$

Hence, the formula $\sup _{t \in \mathbb{R}}|\sin (t)||\cos (t)|=\frac{1}{2}$ gives

$$
\prod_{j=0}^{1} \sup _{s \in[\underline{\tau}, \bar{\tau}]} \rho(t-j s) \leq \frac{81}{64}\left[\frac{1}{2}+\frac{1}{10}\right]^{2}=\frac{729}{1600}
$$

for all $t \geq 0$. Thus (4) is satisfied with $p=1$.

\section{ILLUSTRATIONS}

\section{A. Relaxed Halanay's Inequality Conditions}

As noted above, Halanay's inequality and its generalizations play an important role in the analysis of delayed and switched systems. This motivates the study of continuous piecewise $C^{1}$ functions $v_{a}:[-T,+\infty) \rightarrow[0,+\infty)$ that admit constants $a>0$ and $T>0$ and bounded piecewise continuous functions $c:[0,+\infty) \rightarrow[0,+\infty)$ such that

$$
\dot{v}_{a}(t) \leq-a v_{a}(t)+c(t)\left|v_{a}\right|_{[t-T, t]}
$$

holds for Lebesgue almost all $t \geq 0$, in cases where the supremum $\bar{c}$ of $c$ is such that $\bar{c}>a>0$ (and which therefore are outside the scope of standard Halanay's inequalities requiring $a>\bar{c}$ ). In this subsection, we provide conditions on $c$ under which functions $v$ that satisfy the preceding conditions also satisfy the requirements of our theorem. We also provide an example that illustrates why the conditions in this subsection are less restrictive than those of [?].

To this end, first notice that, since (9) implies that

$$
\dot{v}_{a}(t) \leq-a v_{a}(t)+\bar{c}\left|v_{a}\right|_{[t-T, t]},
$$

we deduce from Lemma C.1 in Appendix C below that for any $p_{*}>0$, the inequality

$$
\left|v_{a}\right|_{[t-T, t]} \leq\left|v_{a}\right|_{\left[t-p_{*}-T, t-p_{*}\right]} e^{(\bar{c}-a)\left(p_{*}+T\right)}
$$

is satisfied for all $t \geq p_{*}$. Choosing $p_{*}=T$ and setting $c_{\triangle}(t)=e^{2(\bar{c}-a) T} c(t)$, we deduce from (9) and (11) that

$$
\dot{v}_{a}(t) \leq-a v_{a}(t)+c_{\triangle}(t)\left|v_{a}\right|_{[t-2 T, t-T]}
$$

for all $t \geq T$. We can apply variation of parameters to (12) on the interval $[t-T, t]$ to get

$$
\begin{aligned}
v_{a}(t) \leq & e^{-a T} v_{a}(t-T) \\
& +\int_{t-T}^{t} e^{a(\ell-t)} c_{\triangle}(\ell)\left|v_{a}\right|_{[\ell-2 T, \ell-T]} \mathrm{d} \ell \\
\leq & e^{-a T} v_{a}(t-T) \\
& +\int_{t-T}^{t} e^{a(\ell-t)} c_{\triangle}(\ell) \mathrm{d} \ell\left|v_{a}\right|_{[t-3 T, t-T]}
\end{aligned}
$$

for all $t \geq 2 T$. Consequently, for all $t \geq 2 T$, we have

$$
\begin{aligned}
& v_{a}(t) \leq \rho_{a}(t)\left|v_{a}\right|_{[t-3 T, t-T]}, \\
& \text { where } \rho_{a}(t)=e^{-a T}+\int_{t-T}^{t} e^{a(\ell-t)} c_{\triangle}(\ell) \mathrm{d} \ell .
\end{aligned}
$$

Now, we can apply our theorem with $\eta=0$. The bound $\rho_{a}(t) \leq \bar{\rho}$ holds for all $t \geq 0$ with the choice

$$
\bar{\rho}=e^{-a T}+\frac{\left(1-e^{-a T}\right) \bar{c}}{a} e^{2(\bar{c}-a) T}
$$

for all $t \geq 2 T$.

As a special case, the requirements of our theorem hold with $a=T=\underline{\tau}=1, \bar{\tau}=3 T=3, p=2$, any $\epsilon \in$ $(0,1), v(t)=v_{a}(t+2 T), \rho(t)=\rho_{a}(t+2 T)$ with $\rho_{a}$ as defined in (14) with the period 9 function $c$ that is defined by $c(t)=\frac{2}{3}$ if $t \in[0,3) \cup[6,9)$ and $c(t)=\frac{21}{16}$ if $t \in[3,6)$, and $\omega=0.654645$. Hence, $v(t)$ converges exponentially to 0 as $t \rightarrow+\infty$. On the other hand, we next show that the preceding example does not satisfy the requirements of the Halanay's inequality generalization from [?, Theorem 1].

To this end, recall that the main sufficient condition for exponential convergence to 0 in [?, Theorem 1] for a continuous and piecewise $C^{1}$ function $w:[-T,+\infty) \rightarrow$ $[0,+\infty)$ is that it satisfies $\dot{w}(s) \leq-a w(s)+\mathcal{L}(s)|w|_{[s-T, s]}$ 
for Lebesgue almost all $s \geq 0$ with constants $a>0$ and $T>0$ and a function $\mathcal{L}$ of the form

$$
\mathcal{L}(t)=\left\{\begin{array}{cl}
\bar{\epsilon}, & \text { if } t \notin E \\
\bar{\varphi}, & \text { if } t \in E
\end{array}\right.
$$

where the set $E$ has the form

$$
E=\cup_{i \in \mathbb{N}}\left[t_{i}, t_{i}+T\right),
$$

the constants $\bar{\epsilon}$ and $\bar{\varphi}$ satisfy $0 \leq \bar{\epsilon}<a \leq \bar{\varphi}$, and the constant $T>0$ is such that $\underline{T}>2 T$ and such that

$$
\bar{\varphi}\left[e^{a(2 T-\underline{T})}+\frac{\bar{\epsilon}}{a}\right] e^{2 T \bar{\varphi}}+\frac{2 T \bar{\varphi}}{\underline{T}}<a,
$$

for any values $t_{i}$ that satisfy $t_{0}=0$ and $\underline{T} \leq t_{i+1}-t_{i} \leq \bar{T}$ for all $i \in \mathbb{Z}_{\geq 0}$ and some constants $\underline{T}>0$ and $\bar{T} \geq \underline{T}$. Applying [?, Theorem 1] to the preceding example would call for choosing $w(s)=v_{a}(s+3), \mathcal{L}(s)=c(s+3), a=$ $1, t_{0}=0, t_{i+1}=t_{i}+9$ for all $i \in \mathbb{Z}_{\geq 0}, T=3, \underline{T}=$ $\bar{T}=9, \bar{\varphi}=21 / 16$, and $\bar{\epsilon}=2 / 3$, but then (18) would not be satisfied because its left side would be $11.8039>a$. Since the preceding example is covered by Theorem 1 but not [?, Theorem 1], this illustrates how Theorem 1 is less conservative than [?, Theorem 1].

\section{B. Systems with Switching Delays}

We next revisit the class of systems with switching delays from [?], under the new and less restrictive generalized Halanay's conditions from the preceding subsection.

Let the sequence $t_{i}$ satisfy the requirements of Section III-A for some $\underline{T}>0$ and $\bar{T} \geq \underline{T}$, and $\tau_{l}$ and $\tau_{s}$ be two constants such that

$$
\underline{T}>5\left(\tau_{l}+\tau_{s}\right)
$$

and $\tau_{l}>\tau_{s} \geq 0$. We study the systems

$$
\dot{x}(t)=M x(t)+N x(t-\tau(t))
$$

where $x$ is valued in $\mathbb{R}^{n}$, and where $\tau$ is a time-varying piecewise continuous unknown delay such that

$$
0 \leq \tau(t) \leq \tau_{s} \text { if } t \notin E \text {, and } 0 \leq \tau(t) \leq \tau_{l} \text { if } t \in E
$$

where $E$ is from (17) for the constant $T=\tau_{s}+\tau_{l}$, and the matrices $M \in \mathbb{R}^{n \times n}$ and $N \in \mathbb{R}^{n \times n}$ are constant for any $n$. Following [?, Section 4.2], we assume:

Assumption 1: There are a symmetric positive definite matrix $Q \in \mathbb{R}^{n \times n}$ and a constant $q>0$ such that

$$
Q(M+N)+(M+N)^{\top} Q \leq-q Q
$$

and $I \leq Q$ are satisfied.

In terms of the notation from Assumption 1 and

$$
L=\frac{2\left|N^{\top} Q N\right|(|M|+|N|)^{2}}{q},
$$

we also use the following, whose proof consists of the first part of the proof of [?, Proposition 1]:

Lemma 1: With the preceding notation and under Assumption 1, the time derivative of the function $U(x)=$ $x^{\top} Q x$ along all solutions of (20) is such that

$$
\dot{U}(t) \leq-\frac{q}{2} U(x(t))+L \tau_{s}^{2} \sup _{m \in\left[t-\tau_{l}-\tau_{s}, t\right]} U(x(m))
$$

for all $t \in[0,+\infty) \backslash E$ and

$$
\dot{U}(t) \leq-\frac{q}{2} U(x(t))+\frac{8\left|N^{\top} Q N\right|}{q} \sup _{l \in\left[t-\tau_{l}, t\right]} U(x(l))
$$

for all $t \in E$.

Using the constant $a_{*}$ and function $b_{*}$ that are defined by $a_{*}=e^{-q\left(\tau_{s}+\tau_{l}\right) / 2}$ and $b_{*}(\ell)=\frac{2\left(1-a_{*}\right) \ell}{q} e^{2(R-q / 2)\left(\tau_{l}+\tau_{s}\right)}$

where $R=8\left|N^{\top} Q N\right| / q$, we prove this consequence of Lemma 1 and Theorem 1 in Appendix D, where (27) can be interpreted to mean that $N$ is small relative to $T$ :

Proposition 1: With the above notation, let Assumption 1 hold, and assume that $L \tau_{s}^{2} \leq q / 2<8\left|N^{\top} Q N\right| / q$ and

$$
\left(a_{*}+b_{*}\left(L \tau_{s}^{2}\right)\right)\left(a_{*}+b_{*}\left(\frac{8\left|N^{\top} Q N\right|}{q}\right)\right)<1 .
$$

Then the origin of (20) is a globally exponentially stable equilibrium point on $\mathbb{R}^{n}$.

\section{Continuous-Discrete System}

We next illustrate Theorem 1 using a continuous-discrete system, for which we derive sufficient conditions for exponential stability that we believe were beyond the scope of previous results. First define a function $\sigma$ by $\sigma(t)=k$ when $t \in[k, k+1)$ for all $k \in \mathbb{Z}_{\geq 0}$. We use the sequence $t_{k}=k$ and any function $\theta$ such that $\theta(i) \in[0, \bar{\theta}]$ for all $i \in \mathbb{Z}_{\geq 0}$ for some constant $\bar{\theta}>1$. Given a constant $\bar{h} \in(0,1)$, let $h:[0,+\infty) \rightarrow[0, \bar{h}]$ be any piecewise continuous function.

Consider the continuous-discrete system

$$
\left\{\begin{aligned}
\dot{x}(t) & =-x(t)+\alpha x(t-h(t)) \\
x\left(t_{\sigma(t)}\right) & =\theta\left(t_{\sigma(t)}\right) x\left(t_{\sigma(t)}^{-}\right)
\end{aligned}\right.
$$

with $x$ valued in $\mathbb{R}$ and $\alpha>0$ being a constant. This is continuous-discrete, because its state is reset at the times $t_{k}$. Our assumptions on the delay $h$ ensure that for each initial function, the solution of (28) is uniquely defined on $[0,+\infty)$. Let us determine functions $\theta$ and constants $\alpha$ for which the origin of (28) is globally exponentially stable on $\mathbb{R}$.

By integrating the first equation of (28), we get

$$
\begin{aligned}
x(t)= & e^{-t+t_{\sigma(t)}} x\left(t_{\sigma(t)}\right) \\
& +\alpha \int_{t_{\sigma(t)}}^{t} e^{-t+m} x(m-h(m)) \mathrm{d} m \\
= & e^{-t+t_{\sigma(t)}} \theta\left(t_{\sigma(t)}\right) x\left(t_{\sigma(t)}^{-}\right) \\
& +\alpha \int_{t_{\sigma(t)}}^{t} e^{-t+m} x(m-h(m)) \mathrm{d} m
\end{aligned}
$$

for all $t \geq 1$. Also, since $t-1 \in[\sigma(t-1), \sigma(t))$, we can integrate the first equation of (28) on $\left(t-1, t_{\sigma(t)}\right)$ to get

$$
\begin{aligned}
& x\left(t_{\sigma(t)}^{-}\right)=e^{-t_{\sigma(t)}+t-1} x(t-1) \\
& +\alpha \int_{t-1}^{t_{\sigma(t)}} e^{-t_{\sigma(t)}+m} x(m-h(m)) \mathrm{d} m .
\end{aligned}
$$

By combining (29) and (30), we obtain

$$
\begin{aligned}
& x(t)=\frac{\theta\left(t_{\sigma(t)}\right)}{e} x(t-1) \\
& +\alpha \theta\left(t_{\sigma(t)}\right) \int_{t-1}^{t_{\sigma(t)}} e^{m-t} x(m-h(m)) \mathrm{d} m \\
& +\alpha \int_{t_{\sigma(t)}}^{t} e^{-t+m} x(m-h(m)) \mathrm{d} m .
\end{aligned}
$$


Consequently,

$$
\begin{aligned}
|x(t)| \leq & \frac{\theta\left(t_{\sigma(t)}\right)}{e}|x(t-1)| \\
& +\alpha\left[\theta\left(t_{\sigma(t)}\right) \int_{t-1}^{t_{\sigma(t)}} e^{m-t} \mathrm{~d} m\right. \\
& \left.+\int_{t_{\sigma(t)}^{t}}^{t} e^{m-t} \mathrm{~d} m\right] \sup _{\ell \in[t-1-\bar{h}, t]}|x(\ell)| \\
\leq & \frac{\theta\left(t_{\sigma(t)}\right)}{e}|x(t-1)| \\
& +\alpha \bar{\theta}\left(1-e^{-1}\right) \sup _{\ell \in[t-1-\bar{h}, t]}|x(\ell)| \\
\leq & \frac{\theta\left(t_{\sigma(t)}\right)}{e}|x(t-1)| \\
& +\alpha \bar{\theta}\left(1-e^{-1}\right) \sup _{\ell \in[t-1-\bar{h}, t-1]}|x(\ell)| \\
& +\alpha \bar{\theta}\left(1-e^{-1}\right) \sup _{\ell \in[t-1, t]}|x(\ell)| \\
\leq & \left\{\frac{\theta\left(t_{\sigma(t)}\right)}{e}+\alpha \bar{\theta}\left(1-\frac{1}{e}\right)\right\} \sup _{\ell \in[t-1-\bar{h}, t-1]}|x(\ell)| \\
& +\alpha \bar{\theta}\left(1-e^{-1}\right) \sup _{\ell \in[t-1, t]}|x(\ell)|
\end{aligned}
$$

for all $t \geq 1$, because $\bar{\theta}>1$. The last inequality in (32) is of the type (6), with $\eta=0$ and the functions $v(t)=|x(t+1)|$ and $\rho(t)=\rho_{0}(t+1)$ with $\rho_{0}(t)$ being the quantity in curly braces in (32). Thus, we can apply Theorem 1.

To illustrate this fact, define $\theta: \mathbb{Z}_{\geq 0} \rightarrow \mathbb{R}$ by

$$
\theta(3 l)=c_{0}, \theta(3 l+1)=c_{1}, \text { and } \theta(3 l+2)=c_{2}
$$

for all $l \in \mathbb{Z}_{\geq 0}$, for constants $c_{0}, c_{1}$, and $c_{2}>1$ such that

$$
c_{1} c_{2}^{2}<e^{3} \text { and } 0 \leq c_{0}<c_{1}<c_{2}<\frac{e}{\alpha(e-1)},
$$

where the last inequality in (34) will be used to ensure that $\alpha \bar{\theta}\left(1-\frac{1}{e}\right)<1$, where we choose $\bar{\theta}=c_{2}$. Let us take $p=2$, $\underline{\tau}=1$, and $\bar{\tau}=1+\bar{h}$, and assume that $\alpha \in[0, \bar{\alpha})$ with

$$
0<\bar{\alpha} \leq \frac{1}{e-1} .
$$

This allows cases where $c_{2}>e$, and therefore also times $t$ when the quantity in curly braces in (32) is strictly larger than 1 . In terms of the constants

$$
c_{i}^{*}=\frac{c_{i}}{e}+\alpha c_{2}\left(1-\frac{1}{e}\right) \text { for } i=1,2,
$$

we can choose the constant $\Omega$ from Theorem 1 to be

$$
\begin{aligned}
\Omega= & c_{1}^{*}\left(c_{2}^{*}\right)^{2}+\alpha\left(1-\frac{1}{e}\right) \sum_{i=0}^{2} c_{2}^{i+1}\left(\frac{1}{e}+\alpha\left(1-\frac{1}{e}\right)\right)^{i} \\
= & \frac{c_{1} c_{2}^{2}}{e^{3}}+\alpha \frac{c_{1} c_{2}^{2}}{e^{2}}\left(1-\frac{1}{e}\right)+\frac{c_{1} c_{2}}{e} \alpha\left(1-\frac{1}{e}\right) c_{2}^{*} \\
& +\alpha c_{2}\left(1-\frac{1}{e}\right)\left[\frac{c_{2}}{e}+\alpha c_{2}\left(1-\frac{1}{e}\right)\right] c_{2}^{*} \\
& +\alpha\left(1-\frac{1}{e}\right) \sum_{i=0}^{2} c_{2}^{i+1}\left(\frac{1}{e}+\alpha\left(1-\frac{1}{e}\right)\right)^{i} .
\end{aligned}
$$

Then, (35) gives

$$
\begin{aligned}
\Omega \leq & \frac{c_{1} c_{2}^{2}}{e^{3}}+\alpha\left(1-\frac{1}{e}\right) c_{2}\left[\frac{c_{1} c_{2}+2 c_{1} c_{2}+4 c_{2}^{2}}{e^{2}}\right. \\
& \left.+\sum_{i=0}^{2}\left(\frac{2 c_{2}}{e}\right)^{i}\right] .
\end{aligned}
$$

Using (34) and the constant

$$
c^{* *}=\frac{3 c_{1} c_{2}+4 c_{2}^{2}}{e^{2}},
$$

we know that there are constants $\bar{\alpha}$ such that

$$
0<\bar{\alpha}<\min \left\{\frac{1}{e-1}, \frac{1-\frac{c_{1} c_{2}^{2}}{e^{3}}}{\left(1-\frac{1}{e}\right) c_{2}\left[c^{* *}+\sum_{i=0}^{2}\left(\frac{2 c_{2}}{e}\right)^{i}\right]}\right\} \text { (40) }
$$

and then $\Omega<1$ when $\alpha \in(0, \bar{\alpha}]$. Then Theorem 1 implies that the origin of the system (28) is exponentially stable.

\section{CONClusions}

We advanced the literature on trajectory-based methods, which are used to prove asymptotic convergence properties for continuous-discrete systems and systems with switched delays which are beyond the scope of traditional Lyapunov function methods. Our main result involves new sup-delay inequalities. While reminiscent of works such as [?], we believe that our sup-delay inequality approach is a novel contribution that can more broadly benefit applied analysis. Compared with earlier trajectory-based methods, the novelty of our innovation was the relaxation of a contractivity requirement. This made it possible to prove asymptotic convergence under less restrictive versions of Halanay's inequality compared to earlier generalized Halanay's inequalities. Our applications to continuous-discrete systems and to systems with switching delays illustrated the value of our new approach. We hope to develop extensions to discretetime inequalities arising from discrete event systems and sampled data control of continuous time systems, and to event-triggered control of transport-reaction equations [?] and integral-delay inequalities in age-structured models [?].

\section{APPENDIX A: MAIN LEMMAS}

The following result from [?, Lemma 1] can be viewed as a contractivity condition because $\kappa \in(0,1)$ :

Lemma A.1: Let $T^{\star}>0$ be a constant. Let $\mu$ : $\left[-T^{\star},+\infty\right) \rightarrow[0,+\infty)$ and $d:[0,+\infty) \rightarrow[0,+\infty)$ be piecewise continuous and locally bounded. Assume that there exists a constant $\kappa \in(0,1)$ such that

$$
\mu(s) \leq \kappa|\mu|_{\left[s-T^{*}, s\right]}+d(s)
$$

holds for all $s \geq 0$. Then the inequality

$$
\mu(s) \leq|\mu|_{\left[s_{0}-T^{\star}, s_{0}\right]} e^{\frac{\ln (\kappa)}{T^{\star}}\left(s-s_{0}\right)}+\frac{1}{1-\kappa}|d|_{\left[s_{0}, s\right]}
$$

holds for all $s \geq s_{0}$ and $s_{0} \geq 0$.

We prove the following (but see Remark A.4-A.5 for discussions of why its assumptions do not imply that $\bar{\rho} \leq 1$ and why we cannot replace $\underline{\tau}$ by 0 in our condition (A.3)):

Lemma A.2: Let $v:[-\bar{\tau},+\infty) \rightarrow[0,+\infty)$ and $\delta:$ $[0,+\infty) \rightarrow[0,+\infty)$ be locally bounded piecewise continuous functions such that

$$
v(t) \leq \rho(t) \sup _{\ell \in[t-\bar{\tau}, t-\underline{\tau}]} v(\ell)+\delta(t)
$$

holds for all $t \geq 0$. Consider the function

$$
\delta_{\star}(t)=\sum_{i=0}^{p} \bar{\rho}^{i}|\delta|_{[t-i \bar{\tau}, t-i \underline{\tau}]} .
$$


Then the following two conclusions hold: (a) the inequality

$$
v(t) \leq \omega|v|_{[t-(p+1) \bar{\tau}, t]}+\delta_{\star}(t)
$$

holds for all $t \geq p \bar{\tau}$ and (b) the inequality

$v(t) \leq|v|_{\left[t_{0}-(p+1) \bar{\tau}, t_{0}\right]} e^{\frac{\ln (\omega)}{(p+1) \bar{\tau}}\left(t-t_{0}\right)}+\frac{|\delta|_{\left[t_{0}-p \bar{\tau}, t\right] \sum_{i=0}^{p} \bar{\rho}^{i}}}{1-\omega}$

holds for all $t \geq t_{0}$ and $t_{0} \geq p \bar{\tau}$.

Proof: From (A.3), we deduce that

$$
|v|_{[t-\bar{\tau}, t-\underline{\tau}]} \leq|\rho|_{[t-\bar{\tau}, t-\underline{\tau}]}|v|_{[t-2 \bar{\tau}, t-2 \underline{\tau}]}+|\delta|_{[t-\bar{\tau}, t-\underline{\tau}]}
$$

for all $t \geq \bar{\tau}$. By induction, one can prove that for all $t \geq p \bar{\tau}$ and any $k \in\{0, \ldots, p\}$, we have

$$
\begin{aligned}
|v|_{[t-k \bar{\tau}, t-k \underline{\tau}]} \leq & |\rho|_{[t-k \bar{\tau}, t-k \underline{\tau}]}|v|_{[t-(k+1) \bar{\tau}, t-(k+1) \underline{\tau}]} \\
& +|\delta|_{[t-k \bar{\tau}, t-k \underline{\tau}]} .
\end{aligned}
$$

To summarize, we have

$$
\begin{aligned}
v(t) & \leq \varsigma_{0}(t)|v|_{[t-\bar{\tau}, t-\underline{\tau}]}+\delta(t) \\
|v|_{[t-\bar{\tau}, t-\underline{\tau}]} \leq \varsigma_{1}(t)|v|_{[t-2 \bar{\tau}, t-2 \underline{\tau}]}+|\delta|_{[t-\bar{\tau}, t-\underline{\tau}]} & \vdots \\
|v|_{[t-p \bar{\tau}, t-p \underline{\tau}]} \leq & \varsigma_{p}(t)|v|_{[t-(p+1) \bar{\tau}, t-(p+1) \underline{\tau}]} \\
& +|\delta|_{[t-p \bar{\tau}, t-p \underline{\tau}]}
\end{aligned}
$$

for all $t \geq p \bar{\tau}$, where $\varsigma_{k}(t)=|\rho|_{[t-k \bar{\tau}, t-k \underline{\tau}]}$ for all $k \in$ $\{1, \ldots, p\}$. Setting $\lambda_{j}(t)=\prod_{i=0}^{j} \varsigma_{i}(t)$ for all integers $j \geq 0$, we have the immediate consequence

$$
\begin{aligned}
v(t) \leq & \varsigma_{0}(t)|v|_{[t-\bar{\tau}, t-\tau]}+\delta(t) \\
\varsigma_{0}(t)|v|_{[t-\bar{\tau}, t-\underline{\tau}] \leq} & \varsigma_{1}(t) \varsigma_{0}(t)|v|_{[t-2 \bar{\tau}, t-2 \underline{\tau}]} \\
& +\varsigma_{0}(t)|\delta|_{[t-\bar{\tau}, t-\underline{\tau}]} \\
\vdots & \\
\lambda_{p-1}(t)|v|_{[t-p \bar{\tau}, t-p \underline{\tau}] \leq} & \lambda_{p}(t)|v|_{[t-(p+1) \bar{\tau}, t-(p+1) \tau]} \\
& +\lambda_{p-1}(t)|\delta|_{[t-p \bar{\tau}, t-p \underline{\tau}]}
\end{aligned}
$$

for all $t \geq p \bar{\tau}$. By moving the left side terms in the last $p$ inequalities in (A.9) to their right sides, and then adding together the $p+1$ inequalities that result, we get

$$
\begin{aligned}
v(t) \leq & \lambda_{p}(t)|v|_{[t-(p+1) \bar{\tau}, t-(p+1) \underline{\tau}]}+\delta(t) \\
& +\sum_{i=1}^{p} \lambda_{i-1}(t)|\delta|_{[t-i \bar{\tau}, t-i \underline{\tau}]} .
\end{aligned}
$$

It follows from (4) that (A.5) holds. Since $\omega \in(0,1)$, we can apply Lemma A.1 with $\mu(s)=v(s+p \bar{\tau})$ and $d(s)=$ $\delta_{*}(s+p \bar{\tau})$, the contractivity constant $\kappa=\omega, T^{*}=(p+1) \bar{\tau}$, and the initial time $s_{0}=t_{0}-p \bar{\tau}$ to obtain part (b).

Remark A.4: The fundamental inequality (4) does not imply that $\bar{\rho} \leq 1$. For instance, it is satisfied for $\underline{\tau}=\bar{\tau}=1$, $p=1, \bar{\rho}=2$ and the period two function $\rho$ defined by $\rho(t)=2$ for all $t \in[i, i+1)$ and even $i \in \mathbb{Z}_{\geq 0}$ and $\rho(t)=\frac{1}{4}$ for all $t \in[i, i+1)$ and odd $i \in \mathbb{Z}_{\geq 0}$. Thus, Lemma A.2 is not a direct consequence of [?, Lemma 1]. See also Remark 3 for a case where $\rho$ is bounded, continuous, and satisfies (4) for all $t \geq p \bar{\tau}$ and some constants $p$, $\underline{\tau}$, and $\bar{\tau}>\underline{\tau}$, but where $\rho$ can take some of its values in $(1,+\infty)$.

Remark A.5: One cannot let $\tau$ be equal to zero, because when $\underline{\tau}=0$, a function $v$ satisfying all the other conditions of Lemma A.2 may be unbounded even when $\delta$ is bounded, as illustrated by the following example. Let $\rho:[0,+\infty) \rightarrow$ $[0,+\infty)$ be a function such that there are intervals $\left[t_{s, i}, t_{r, i}\right]$ with $t_{r, i}>t_{s, i}>0$ and $\lim _{i \rightarrow+\infty} t_{s, i}=+\infty$ such that $\rho(t) \geq 1$ for all $t \in\left[t_{s, i}, t_{r, i}\right]$. Then, for any constant $\bar{\tau}>0$, the function $v:[-\bar{\tau},+\infty) \rightarrow[0,+\infty)$ defined by $v(t)=0$ when $t \notin \cup_{i \in \mathbb{N}}\left[t_{s, i}, t_{r, i}\right]$ and $v(t)=i$ when $t \in\left[t_{s, i}, t_{r, i}\right]$ is a piecewise continuous function such that $v(t) \leq \rho(t) \sup _{\ell \in[t-\bar{\tau}, t]} v(\ell)$ for all $t \geq 0$ and $v$ is unbounded.

\section{APPEndix B: Proof OF TheOrem 1}

We use the simplifying notation

$$
\delta_{\star}(t)=\sum_{i=0}^{p} \bar{\rho}^{i}|\delta|_{[t-i \bar{\tau}, t-i \underline{\tau}]},
$$

where $\delta(t)=\epsilon|v|_{[t-\underline{\tau}, t]}+\eta(t)$. By applying Lemma A.2, it follows from (A.5) that

$$
v(t) \leq \omega|v|_{[t-(p+1) \bar{\tau}, t]}+\delta_{\star}(t)
$$

for all $t \geq p \bar{\tau}$. Consequently,

$$
\begin{aligned}
v(t) \leq & \omega|v|_{[t-(p+1) \bar{\tau}, t]}+\epsilon \sum_{i=0}^{p} \bar{\rho}^{i}|v|_{[t-p \bar{\tau}-\underline{\tau}, t]} \\
& +\sum_{i=0}^{p} \bar{\rho}^{i}|\eta|_{[t-p \bar{\tau}, t]}
\end{aligned}
$$

for all $t \geq p \bar{\tau}$. Since $\underline{\tau} \leq \bar{\tau}$, the inequality

$$
v(t) \leq \Omega|v|_{[t-(p+1) \bar{\tau}, t]}+\sum_{i=0}^{p} \bar{\rho}^{i}|\eta|_{[t-p \bar{\tau}, t]}
$$

therefore holds for all $t \geq p \bar{\tau}$. Since $\Omega \in(0,1)$, it follows from applying Lemma A. 1 to $\mu(s)=v(s+p \bar{\tau})$ that (7) holds for all $t \geq t_{0}$ and for all $t_{0} \geq p \bar{\tau}$.

\section{APPEndiX C: Unstable Halanay's InEQUALity}

We used this lemma in our analysis of the generalized Halanay's conditions in Section III-A for cases where the coefficient of the gain term can exceed the decay rate:

Lemma C.1: Let $T>0$ be a constant and $v$ : $[-T,+\infty) \rightarrow[0,+\infty)$ be a continuous and piecewise $C^{1}$ function that admits constants $a>0$ and $c>a$ such that

$$
\dot{v}(t) \leq-a v(t)+c \sup _{\ell \in[t-T, t]} v(\ell)
$$

holds for Lebesgue almost all $t \geq 0$. Then for any constants $p_{*}>0$ and $t_{0} \geq p_{*}$, the inequality

$$
|v|_{\left[t_{0}-T, t_{0}\right]} \leq|v|_{\left[t_{0}-p_{*}-T, t_{0}-p_{*}\right]} e^{(c-a)\left(p_{*}+T\right)}
$$

holds.

Proof: Given any constant $\epsilon>0$, let

$$
b\left(t_{0}\right)=|v|_{\left[t_{0}-T, t_{0}\right]}+\epsilon \text { and } s(t)=b\left(t_{0}\right) e^{(c-a)\left(t-t_{0}+T\right)} .
$$

Then $\dot{s}(t)=-a s(t)+c s(t)$ for all $t \geq 0$. Since $s$ is nondecreasing, it follows that $\tilde{e}(t)=v(t)-s(t)$ satisfies

$$
\begin{aligned}
\dot{\tilde{e}}(t) & \leq-a \tilde{e}(t)+c\left(\sup _{\ell \in[t-T, t]} v(\ell)-s(t)\right) \\
& \leq-a \tilde{e}(t)+c \sup _{\ell \in[t-T, t]} \tilde{e}(\ell)
\end{aligned}
$$


for Lebesgue almost all $t \geq 0$. Note too that we have

$$
\begin{aligned}
\tilde{e}(m) \leq & |v|_{\left[t_{0}-T, m\right]}-b\left(t_{0}\right) e^{(c-a)\left(m-t_{0}+T\right)} \\
& =|v|_{\left[t_{0}-T, t_{0}\right]} \\
& \leq-\left(|v|_{\left[t_{0}-T, t_{0}\right]}+\epsilon\right) e^{(c-a)\left(m-t_{0}+T\right)} \\
\leq & -\epsilon
\end{aligned}
$$

for all $m \in\left[t_{0}-T, t_{0}\right]$, and therefore also a value $t_{0}^{\sharp}>t_{0}$ such that $\tilde{e}(m) \leq-\epsilon / 2$ for all $m \in\left[t_{0}-T, t_{0}^{\sharp}\right]$ (since $\tilde{e}$ is continuous). We next show that $\tilde{e}(t) \leq-\epsilon / 2$ for all $t \geq$ $t_{0}-T$, by arguing by contradiction. To this end, suppose (for the sake of obtaining a contradiction) that $\tilde{e}(m)>-\epsilon / 2$ for some $m \geq t_{0}-T$. Then, there is a $t_{c}>t_{0}$ such that $\tilde{e}(m) \leq-\epsilon / 2$ when $m \in\left[t_{0}, t_{c}\right)$ and $\tilde{e}\left(t_{c}\right)=-\epsilon / 2$. Pick any constant $\delta_{0} \in(0,(c-a) \epsilon / 2)$. Then we can use (C.3) and the continuity of $\tilde{e}$ to find a constant $\epsilon_{0}>0$ such that

$$
\begin{aligned}
\dot{\tilde{e}}(r) & \leq-a \tilde{e}(r)+c \sup _{\ell \in[r-T, r]} \tilde{e}(\ell) \\
& \leq a \epsilon / 2+c \sup _{\ell \in[r-T, r]} \tilde{e}(\ell)+\delta_{0} \\
& \leq a \epsilon / 2-c \epsilon / 2+\delta_{0}<0
\end{aligned}
$$

for Lebesgue almost all $r \in\left[t_{c}-\epsilon_{0}, t_{c}\right]$. Hence, there is a $t_{n} \in\left[t_{0}, t_{c}\right)$ such that $\tilde{e}\left(t_{n}\right)>-\epsilon / 2$. This yields a contradiction. Hence, for all $t \geq t_{0}-T$, we have $\tilde{e}(t)<0$ and so also $v(t)<s(t)$. Since $\epsilon$ is arbitrary, we deduce that

$$
v(m) \leq|v|_{\left[t_{0}-T, t_{0}\right]} e^{(c-a)\left(m-t_{0}+T\right)}
$$

for all $m \geq t_{0}-T$.

Thus, for all $t \geq t_{0}$ and for all $m \in[t-T, t]$, we have $v(m) \leq|v|_{\left[t_{0}-T, t_{0}\right]} e^{(c-a)\left(t-t_{0}+T\right)}$. Therefore,

$$
|v|_{[t-T, t]} \leq|v|_{\left[t_{0}-T, t_{0}\right]} e^{(c-a)\left(t-t_{0}+T\right)} .
$$

We can replace $t_{0}$ by $t-p_{*}$ in (C.6) to get

$$
|v|_{[t-T, t]} \leq|v|_{\left[t_{0}-p_{*}-T, t-p_{*}\right]} e^{(c-a)\left(p_{*}+T\right)}
$$

when $t \geq t_{0} \geq p_{*}$, which gives the conclusion when specialized to the case $t=t_{0}$.

\section{APPENDiX D: Proof of Proposition 1}

Along each solution $x:\left[-\tau_{l}-\tau_{s},+\infty\right) \rightarrow \mathbb{R}^{n}$ of (20), we apply the Halanay's inequality analysis from Section III-A with $v_{0}(t)=U(t)=x^{\top}(t) Q x(t), a=q / 2$, and

$$
c(t)= \begin{cases}L \tau_{s}^{2}, & t \in[0,+\infty) \backslash E \\ \frac{8\left|N^{\top} Q N\right|}{q}, & t \in E .\end{cases}
$$

By our choice of $T=\tau_{s}+\tau_{l}$, it follows that if $t \in E$, then $[t-3 T, t-T] \cap E=\emptyset$, because $3 T<\underline{T}-T$. Hence, by the analysis that led to (14) with $\bar{c}=8\left|N^{\top} Q N\right| / q$ and $a=q / 2$, our sufficient condition (4) from Lemma A.2 holds using

$$
\underline{\tau}=\tau_{s}+\tau_{l}, \bar{\tau}=3\left(\tau_{s}+\tau_{l}\right), \text { and } p=1,
$$

because in this case, we can check (by separately considering the cases $t \in E$ and $t \notin E$ ) that the product on the left side of (4) for each $t \geq 0$ is bounded above by

$$
\begin{aligned}
& \rho(t)|\rho|_{[t-3 T, t-T]} \leq \\
& \left(a_{*}+b_{*}\left(L \tau_{s}^{2}\right)\right)\left(a_{*}+b_{*}\left((8 / q)\left|N^{\top} Q N\right|\right)\right)
\end{aligned}
$$

and because the right side of (D.3) is valued in $(0,1)$ by (27). 